\title{
Non-tidal vertical variations and the past star catalogs
}

\author{
Z. X. Li \\ Shanghai Astronomical Observatory, Shanghai 200030, China \\ email: shaolzx@yahoo.com
}

\begin{abstract}
Tidal vertical variations have been removed in astrometric observations, but the non-tidal ones remain unknown. From the repeated observations of gravimetric networks in China, the non-tidal vertical variations at Beijing-Tangshan and West Yunnan are determined, which are of the order of 0"' 2. Astrometric results, including past star catalogs, based on these observations should be corrected.
\end{abstract}

Keywords. astrometry, catalogs

Are there non-tidal vertical variations on Earth? It is an important problem in astronomy since ground optical observations are related to the local vertical (IAU, 1991). Until now only tidal vertical variations are removed in the observations while the non-tidal ones remain unknown. Past star catalogs are still suffering from the uncorrected nontidal vertical variations. It is one of the reasons why the problem of non-tidal vertical variations could not be solved by astrometric techniques while these contaminated star catalogs were being used. Gravimetric technique is able to determine it but repeated gravity measurements performed on a network are needed. In China two gravimetric networks, located in Beijing-Tangshan and West Yunnan, were established in the 1980s, and gravity measurements with high precision are being performed 2 to 4 times per year ever since. Our study has demonstrated the possibility of using these gravimetric observations to determine non-tidal vertical variations (Plumb Line Variations: PLV) there (Li et al., 2005). Tangshan $\left(118.6^{\circ} \mathrm{E}, 39.7^{\circ} \mathrm{N}\right)$ and Midu $\left(100.4^{\circ} \mathrm{E}, 25.3^{\circ} \mathrm{N}\right)$ have been chosen as the two sample sites where the PLV are determined. Figures 1 and 2 show the results.
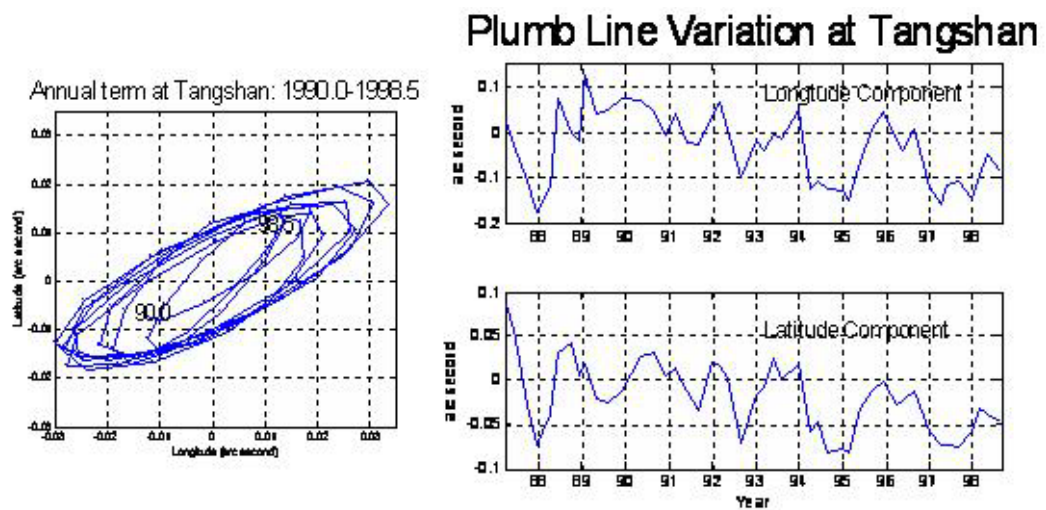

Figure 1. PLV at Tangshan: Annual term (left); PLV (after removing annual term)

It can be seen that the annual term of the PLV at these two sites is of the order of 0." 02-0." 03, while the PLV (after removing annual terms) is of the order of 0.' 2. In Fig.3, it appears that the PLV at Tangshan is related to the earthquakes around ("PLV main 


\section{Plumb Line Variation at Midu}
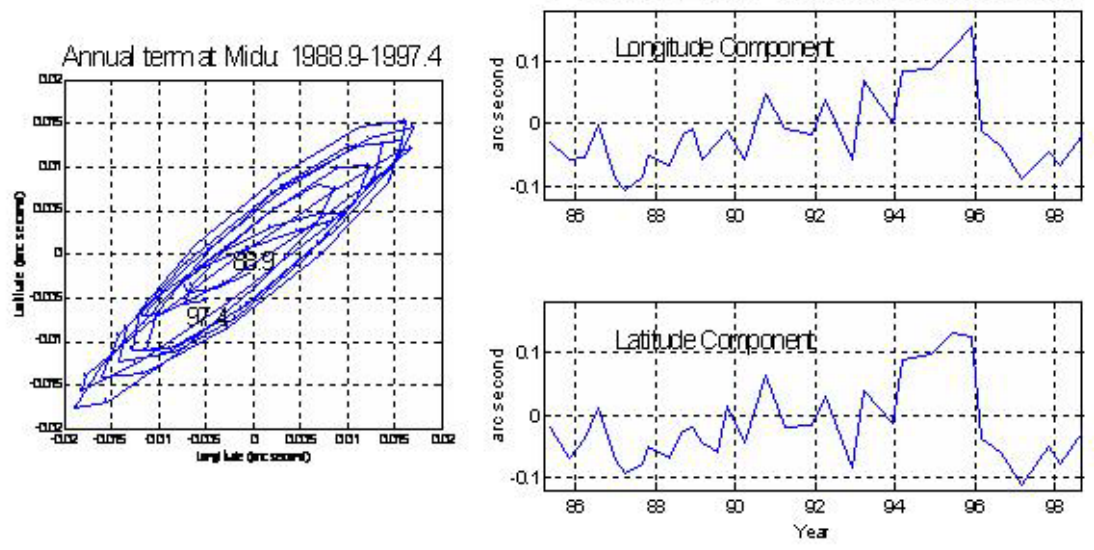

Figure 2. PLV at Midu: Annual term (left); PLV (after removing annual term)

Comparison between PLV main component and earthquakes

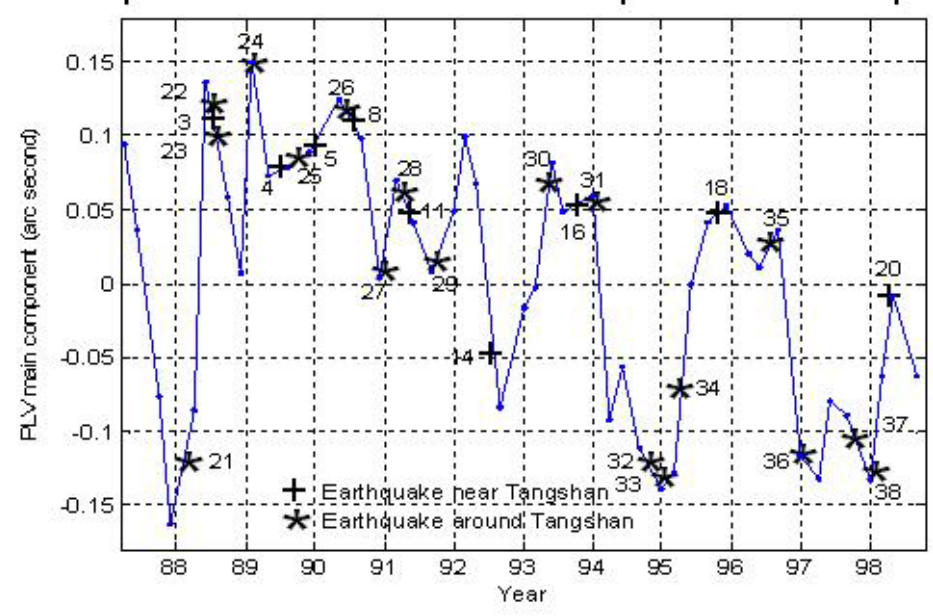

Figure 3. Comparison between the PLV at Tangshan and the earthquakes during 1987-1998

component" is the PLV component in the direction of azimuth of $66.5^{\circ}$, while larger PLV components are shown). Similar phenomenon has also been found in the case of Midu PLV at West Yunnan.

Beijing and Yunnan observatories are located in or near these two regions, but in the past the non-tidal vertical variations (PLV) have never been considered. They exist in the past observations and in the star catalogs based on these uncorrected observations.

\section{References}

IAU 1991, Trans Int Astronomical Union, XXIB, 209

Li, Z. X., Li, H., \& Han, Y. B. 2005, Journal of Geodesy, 78, 593 